\title{
Valproic acid and its inhibition of tumor growth in systemic malignancies: beyond gliomas
}

\author{
Shailendra Kapoor
}

Received: 11 December 2012/Accepted: 31 March 2013/Published online: 16 April 2013

(C) Springer Science+Business Media New York 2013

To the editor,

The recent article by Chen et al. [1] provided for highly stimulating reading. Valproic acid mitigates tumor growth in a number of systemic malignancies besides gliomas.

Similar effects are seen in prostate carcinomas. Valproic acid causes augmentation of p27 levels leading to cell cycle arrest in the cancerous cells [2]. The expression of androgen receptors by the malignant tumor is also decreased. At the same time E-cadherin expression is augmented markedly [3]. Angiogenesis is also inhibited at the same time thus decreasing tumor growth. Cyclin D1 expression is also attenuated at the same time, while cytokeratin-18 expression is increased [4]. This results in a decrease in tumor invasiveness as well as a decline in cancer cell migration. Valproic acid also has an augmenting effect on the radio-sensitivity of prostate carcinomas [5]. It mediates this effect via acetylated p53 dependent modulation of mitochondrial membrane potential in the cancerous cells. Similar effects have been seen in bladder carcinomas. It mediates its anti-neoplastic effect in bladder tumors by inhibiting histone deacetylase [6]. Simultaneous up-regulation of thrombospondin-1 expression is also seen in bladder carcinomas.

Similarly, valproic acid inhibits tumor growth in colorectal malignancies. These effects are especially seen when it is used in combination with IIF. The dual combination enhances Bax expression while Bcl-2 expression is markedly reduced [7]. TIMP-1 activity is also enhanced at the same time, while MMP2 activity is considerably reduced.

S. Kapoor $(\bowtie)$

74 Crossing Place, Mechanicsville, VA, USA

e-mail: shailendrakapoor@yahoo.com
These effects are time dependent. The combination also markedly up-regulates the expression of $\operatorname{RXR} \gamma$. Histone deacetylase activity is also markedly reduced. Valproic acid also augments the radio-sensitivity of colon carcinomas. p53 plays a major role in this valproic acid mediated radio-sensitization. It is clear from the above examples that valproic acid may have significant tumor attenuating effects. There is an urgent need to fully as well as further evaluate these anti-neoplastic effects.

Conflict of interest None.

\section{References}

1. Chen Y, Tsai Y-H, Tseng S-H (2012) Valproic acid affected the survival and invasiveness of human glioma cells through diverse mechanisms. J Neurooncol 109:23-33

2. Sidana A, Wang M, Shabbeer S et al (2012) Mechanism of growth inhibition of prostate cancer xenografts by valproic Acid. J Biomed Biotechnol 2012:180363

3. Zhang L, Wang G, Wang L, Song C, Wang X, Kang J (2011) Valproic acid inhibits prostate cancer cell migration by upregulating E-cadherin expression. Pharmazie 66:614-618

4. Chen X, Wong JY, Wong P, Radany EH (2011) Low-dose valproic acid enhances radiosensitivity of prostate cancer through acetylated p53-dependent modulation of mitochondrial membrane potential and apoptosis. Mol Cancer Res 9:448-461

5. Byler TK, Leocadio D, Shapiro O et al (2012) Valproic acid decreases urothelial cancer cell proliferation and induces thrombospondin-1 expression. BMC Urol 12:21

6. Papi A, Ferreri AM, Guerra F, Orlandi M (2012) Anti-invasive effects and proapoptotic activity induction by the rexinoid IIF and valproic acid in combination on colon cancer cell lines. Anticancer Res 32:2855-2862

7. Chen X, Wong P, Radany E, Wong JY (2009) HDAC inhibitor, valproic acid, induces p53-dependent radiosensitization of colon cancer cells. Cancer Biother Radiopharm 24:689-699 